\title{
Correction to: 45-Year-Old Man with Leg Pain and Numbness
}

Robert Fuino and Waqar Waheed

\section{Correction to: Chapter 32 in T. Malik (ed.), Practical Chronic Pain Management, https://doi.org/10.1007/978-3-030-46675-6_32}

The content of the chapter 32 has been changed. In the originally published version, chapters 32 and 40 were duplicates of the same chapter. The former chapter 32 has now been replaced with a new chapter entitled "45-Year-Old Man with Leg Pain and Numbness."

The updated version of this chapter can be found at https://doi.org/10.1007/978-3-030-46675-6_32 